\title{
Sedimentology and stratigraphy at the rocky coast of the upper Burdigalian Molasse Sea: a case study from Gurlarn near Passau (SE Germany)
}

Dorothea Frieling • Martina Pippèrr •

Simon Schneider $\cdot$ Bettina Reichenbacher

Published online: 5 November 2008

(C) Springer-Verlag 2008

Erratum to: Facies

DOI 10.1007/s10347-008-0161-5

The original version of this article unfortunately contained a mistake. In Table 4 the fifth name of species Lenticulina ottnangensis melvilli (Cushman and Renz) has to be replaced by Lenticulina melvilli (Cushman and Renz). The correct version of Table 4 is given below.

The online version of the original article can be found under doi: 10.1007/s10347-008-0161-5.

D. Frieling $(\bowtie) \cdot$ M. Pippèrr $\cdot$ B. Reichenbacher

Department of Earth and Environmental Sciences,

Section Palaeontology, Ludwig-Maximilians University

of Munich, Richard-Wagner-Str. 10, 80333 Munich, Germany

e-mail: d.frieling@1rz.uni-muenchen.de

S. Schneider

Bayerische Staatssammlung für Paläontologie und Geologie,

Richard-Wagner-Str. 10, 80333 Munich, Germany 
Table 4 Distribution and abundance of benthic foraminifera in the samples G1-G8, Ga, Gb and Gs from the Upper Marine Molasse deposits from the Gurlarn clay pit

\begin{tabular}{|c|c|c|c|c|c|c|c|c|c|c|}
\hline \multicolumn{2}{|l|}{ I } & \multicolumn{3}{|l|}{ II } & \multicolumn{2}{|l|}{ III } & \multirow{2}{*}{$\begin{array}{l}\text { IV } \\
\text { G2 }\end{array}$} & \multicolumn{2}{|l|}{$\mathrm{V}$} & \multirow[t]{2}{*}{ Samples Gurlarn } \\
\hline $\mathrm{Ga}$ & $\mathrm{Gb}$ & G4 & G1 & G5 & Gs & G6 & & G7 & G8 & \\
\hline $\mathrm{b}$ & & & & & & & & & & Textularia gramen d' Orb. \\
\hline $\mathrm{c}$ & $\mathrm{a}$ & & & a & & & & & & Spiroplectammina pectinata (Reuss) \\
\hline $\mathrm{a}$ & $\mathrm{b}$ & & & & & & & & & Sigmoilopsis ottnangensis ${ }^{\mathrm{e}}$ Cicha, Ctyroká and Zapletalova \\
\hline $\mathrm{d}$ & d & $\mathrm{c}$ & $\mathrm{b}$ & $\mathrm{c}$ & a & a & a & $\mathrm{a}$ & & $\begin{array}{l}\text { Lenticulina inornata }(\mathrm{d} \text { ' Orb.)/Lenticulina melvilli } \\
\quad(\text { Cushman and Renz })\end{array}$ \\
\hline $\mathrm{a}$ & $\mathrm{b}$ & & & & & & & & & Lenticulina paupercula (Reuss) \\
\hline $\mathrm{b}$ & & & & & & & & & & Lenticulina vortex (Fichtel and Moll) \\
\hline $\mathrm{b}$ & & & & & & & & & & Astacolus crepidulus (Fichtel and Moll) \\
\hline $\mathrm{a}$ & & & & & & & & & & Marginulina wengeri Rupp and Haunold-Jenke \\
\hline a & & & & & & & & & & Frondicularia sp. \\
\hline $\mathrm{c}$ & $\mathrm{a}$ & $\mathrm{b}$ & a & & & & & & & Polymorphinidae div. sp. \\
\hline $\mathrm{a}$ & $\mathrm{a}$ & $\mathrm{b}$ & & $\mathrm{a}$ & & & & & & Fursenkoina acuta (d' Orb.) \\
\hline a & & & & & & & & & & Uvigerina cf. posthantkeni Papp \\
\hline a & & $\mathrm{b}$ & & & & & & & & Amphicoryna ottnangensis $^{\mathrm{e}}$ (Toula) \\
\hline $\mathrm{b}$ & & & & & & & & $\mathrm{a}$ & & Siphonodosaria aff. verneuili (d' Orb.) \\
\hline $\mathrm{b}$ & $\mathrm{b}$ & & & & & & & & & Elphidium crispum (Linné) \\
\hline $\mathrm{b}$ & & & & & & & & & & Elphidium flexuosum (d' Orb.) \\
\hline $\mathrm{d}$ & $\mathrm{d}$ & $\mathrm{c}$ & $\mathrm{b}$ & $\mathrm{a}$ & $\mathrm{a}$ & $\mathrm{b}$ & $\mathrm{c}$ & & & Elphidium subtypicum Papp \\
\hline $\mathrm{a}$ & & $\mathrm{b}$ & & & & & & & & Elphidium macellum (Fichtel and Moll) \\
\hline a & $\mathrm{b}$ & & & $\mathrm{b}$ & & & & & & Elphidium fichtellianum (d' Orb.) \\
\hline $\mathrm{b}$ & $\mathrm{a}$ & & & & & & $\mathrm{b}$ & & $\mathrm{a}$ & Elphidium cf. hauerinum (d' Orb.) \\
\hline $\mathrm{c}$ & $\mathrm{c}$ & $\mathrm{c}$ & & & & $\mathrm{b}$ & $\mathrm{c}$ & a & & Elphidium glabratum Cushman \\
\hline $\mathrm{b}$ & $\mathrm{c}$ & $\mathrm{c}$ & $\mathrm{b}$ & & & $\mathrm{b}$ & $\mathrm{c}$ & & & Elphidium div. sp. \\
\hline $\mathrm{b}$ & $\mathrm{c}$ & $\mathrm{c}$ & $\mathrm{b}$ & $\mathrm{a}$ & & $\mathrm{b}$ & $\mathrm{a}$ & $\mathrm{a}$ & & Elphidiella minuta (Reuss) \\
\hline $\mathrm{c}$ & $\mathrm{c}$ & $\mathrm{d}$ & c & $\mathrm{a}$ & $\mathrm{a}$ & $\mathrm{b}$ & $\mathrm{c}$ & $\mathrm{b}$ & & Nonion commune (d' Orb.) \\
\hline $\mathrm{c}$ & $\mathrm{b}$ & $\mathrm{a}$ & & & & & & & & Pullenia bulloides (d' Orb.) \\
\hline $\mathrm{b}$ & & & & & & & & & & Pullenia quinqueloba (Reuss) \\
\hline $\mathrm{b}$ & $\mathrm{b}$ & & & & & & & & & Astrononion perfossum (Clodius) \\
\hline $\mathrm{d}$ & $\mathrm{d}$ & $\mathrm{d}$ & $\mathrm{c}$ & $\mathrm{c}$ & $\mathrm{b}$ & $\mathrm{d}$ & $\mathrm{d}$ & $\mathrm{b}$ & $\mathrm{c}$ & Ammonia beccarii (Linné) s.l. \\
\hline $\mathrm{c}$ & & & & & & & & & & Rosalina obtusa d' Orb. \\
\hline $\mathrm{a}$ & & $\mathrm{a}$ & & & & & & & $\mathrm{a}$ & Biapertorbis biaperturatus Pokorny \\
\hline $\mathrm{a}$ & & & & & & & & & & Cancris auriculus (Fichtel and Moll) \\
\hline a & $\mathrm{b}$ & & & & & & & & & Alabamina tangentialis (Clodius) \\
\hline $\mathrm{d}$ & $\mathrm{d}$ & $\mathrm{d}$ & $\mathrm{c}$ & $\mathrm{b}$ & $\mathrm{a}$ & $\mathrm{a}$ & $\mathrm{c}$ & & $\mathrm{b}$ & Cibicididae div. sp. \\
\hline $\mathrm{c}$ & $\mathrm{a}$ & & & & & & & & & Heterolepa dutemplei (d' Orb.) \\
\hline \multirow[t]{10}{*}{$\mathrm{b}$} & $\mathrm{d}$ & $\mathrm{d}$ & $\mathrm{a}$ & $\mathrm{a}$ & & a & $\mathrm{b}$ & & $\mathrm{a}$ & Hanzawaia boueana (d' Orb.) \\
\hline & $\mathrm{a}$ & & & & & & & & & Triloculina gibba d' Orb. \\
\hline & $\mathrm{a}$ & & & & & & & & & Sigmoilinita tenuis (Czjzek) \\
\hline & $\mathrm{b}$ & & & & & $\mathrm{a}$ & & & & $\begin{array}{l}\text { Laevidentalina elegans (d' Orb.) (Gb); } \\
\quad \text { Laevidentalina communis (d' Orb.) (G6) }\end{array}$ \\
\hline & $\mathrm{a}$ & $\mathrm{b}$ & & & & $\mathrm{a}$ & $\mathrm{a}$ & & & Lagena div. sp. \\
\hline & $\mathrm{a}$ & & & $\mathrm{a}$ & & & a & & & Bulimina schischkinskayae Samoilova \\
\hline & $\mathrm{a}$ & & & & & & & & & Trifarina bradyi Cushman \\
\hline & a & & & & & & & & & Oolina globosa (Montagu) \\
\hline & $\mathrm{b}$ & & & & & & & & & Elphidium angulatum (Egger) \\
\hline & a & a & & & & & $\mathrm{b}$ & & $\mathrm{a}$ & "Elphidium" rugulosum Cushman and Wickenden \\
\hline
\end{tabular}


Table 4 continued

\begin{tabular}{|c|c|c|c|c|c|c|c|c|c|c|}
\hline \multicolumn{2}{|l|}{ I } & \multicolumn{3}{|l|}{ II } & \multicolumn{2}{|l|}{ III } & \multirow{2}{*}{$\begin{array}{l}\text { IV } \\
\text { G2 }\end{array}$} & \multicolumn{2}{|l|}{$\mathrm{V}$} & \multirow[t]{2}{*}{ Samples Gurlarn } \\
\hline $\mathrm{Ga}$ & $\mathrm{Gb}$ & G4 & G1 & G5 & Gs & G6 & & G7 & G8 & \\
\hline & $\mathrm{b}$ & $\mathrm{b}$ & & & & & $\mathrm{c}$ & a & a & Elphidiella heteropora (Egger) \\
\hline & $\mathrm{a}$ & $\mathrm{b}$ & a & $\mathrm{a}$ & a & & & & & Elphidiella semiincisa Wenger \\
\hline & $\mathrm{b}$ & & a & & & & & & & Porosononion granosum (d' Orb.) \\
\hline & a & & & & & & & & & Melonis pompilioides (Fichtel and Moll) \\
\hline & $\mathrm{b}$ & & & & & & & & & Asterigerinata planorbis (d' Orb.) \\
\hline & & a & & & & & & & & Saracenaria arcuata (d' Orb.) \\
\hline & & a & & & & & & & & Amphimorphina haueriana Neugeboren \\
\hline & & $\mathrm{a}$ & & & & & & & & Fissurina sp. \\
\hline & & a & & & & & & & & Stilostomella adolphina (d' Orb.) \\
\hline & & $\mathrm{b}$ & & & & & $\mathrm{b}$ & & & Stilostomella perscripta (Egger) \\
\hline & & a & & & & $\mathrm{a}$ & & & & Protelphidium roemeri (Cushman) \\
\hline & & & & $\mathrm{b}$ & & a & a & $\mathrm{c}$ & $\mathrm{b}$ & $\begin{array}{l}\text { Bolivina dilatata Reuss (G5, G6), } \\
\quad \text { Bolivina scitula }{ }^{\mathrm{e}} \text { Hofmann }(\mathrm{G} 2, \mathrm{G} 7, \mathrm{G} 8)\end{array}$ \\
\hline & & & & & & a & & & & Amphicoryna sp. \\
\hline & & & & & & a & $\mathrm{b}$ & & & Elphidium matzenense Papp \\
\hline & & & & & & & $\mathrm{b}$ & & $\mathrm{a}$ & Pappina primiformis $^{\mathrm{e}}$ (Papp and Turnovsky) \\
\hline & & & & & & & $\mathrm{b}$ & & & Pappina breviformis ${ }^{\mathrm{e}}$ (Papp and Turnovsky) \\
\hline & & & & & & & $\mathrm{a}$ & & & Gyroidinoides parvus (Cushman and Renz) \\
\hline & & & & & & & & & $\mathrm{b}$ & Fursenkoina aff. mustoni (Andreae) \\
\hline
\end{tabular}

Some species are lumped: Polymorphinidae = Globulina div. sp. Guttulina div. sp.; Cibicididae = Cibicidoides lopjanicus (Myatlyuk), Cibicidoides tenellus (Ruess), Cibicides lobatulus (Walker and Jacob), Cibicidoides sp.; Lagena div. sp. = Lagena striata (d'orb.), Lagena sulcata (Walker and Jacob), Lagena laevis (Montagu)

a 1 Ex.

b $2-5 \mathrm{Ex}$.

c $6-20$ Ex.

d $>20$ Ex.

e Index fossils (see Table 5) 